INPLASY

To cite: $X u$ et al. Sound therapy for the treatment of chronic subjective tinnitus: $A$ Bayesian network meta analysis of RCTs. Inplasy protocol 202040033. doi: 10.37766/inplasy2020.4.0033

Received: 06 April 2020

Published: 06 April 2020

Corresponding author:

Piao Xu

xuyigenl@163.com

Author Affiliation:

The Third Affiliated Hospital of

Southern Medical

Support: None

Review Stage at time of this submission: Preliminary searches.

Conflicts of interest:

None.

\section{Sound therapy for the treatment of chronic subjective tinnitus:A Bayesian network meta analysis of RCTs}

Xu P1; Li DF²; Zhao HY3; Yang JY4; Lin QP5; Tian GY6.

Review question / Objective: This study evaluated the effectiveness of different kinds of sound in the treatment of tinnitus.

Condition being studied: Tinnitus is a common chronic condition defined as the perception of sound without an auditory stimuli.

Information sources: Chinese language databases included Wanfang data, CNKI database, and VIP Chinese Science and Technique Journals database. English language databases included PubMed, Web of Science, Embase and Cochrane Library.

INPLASY registration number: This protocol was registered with the International Platform of Registered Systematic Review and Meta-Analysis Protocols (INPLASY) on 06 April 2020 and was last updated on 06 April 2020 (registration number INPLASY202040033.

\section{INTRODUCTION}

Review question / Objective: This study evaluated the effectiveness of different kinds of sound in the treatment of tinnitus.

Condition being studied: Tinnitus is a common chronic condition defined as the perception of sound without an auditory stimuli.

\section{METHODS}

Participant or population: Adult patients, aged 18 years or older with tinnitus listed as the primary reason for treatment, were included.

Intervention: Sound therapy was the primary intervention of interest but it may comes in many forms. Therapies that 
combined CBT with any form of audiological intervention were excluded to avoid confounders.

Comparator: RCT comparing various forms of sound therapy to one another were included.

Study designs to be included: Randomized controlled trial.

Eligibility criteria: (i) study focused on patients with tinnitus, (ii) compared the improvement in validated tinnitus specific health-related quality of life (HRQOL) instruments in different kinds of sound therapy, and (iii) written in English or Chinese.

Information sources: Chinese language databases included Wanfang data, CNKI database, and VIP Chinese Science and Technique Journals database. English language databases included PubMed, Web of Science, Embase and Cochrane Library.

Main outcome(s): The primary outcome in this review was improvement in validated tinnitus specific health-related quality of life (HRQOL) instruments.

Quality assessment / Risk of bias analysis: Any risk of bias with the potential of affecting cumulative evidence was considered.Risk of bias assessment, as guided by the Cochrane risk-of bias tool, was performed on all included studies by all three authors.The Grades of Recommendation, Assessment, Development and Evaluation (GRADE) Working Group's system for evaluating the quality of evidence for outcomes reported in systematic reviews and network metaanalysis was completed.

Strategy of data synthesis: Several analyses were performed on the extracted data. First,a qualitative analysis of the studies was undertaken, describing the study, the population, and the methodological characteristics.Second, a NMA comparing the effects of all forms of sound therapies in tinnitus, as this allowed us to compare the direct and indirect relationships between therapies. Treatment effects for all three outcome measures were presented by network diagrams and interval plots indicating SMDs with $95 \% \mathrm{Cl}$. From the NMA results, rankograms were also created for each outcome measure, indicating the probability of each intervention being effective compared to other therapies. Additionally, the inconsistency between direct and indirect results was assessed using inconsistency plots. The plots calculated both inconsistency factors and heterogeneity variance. STATA (StatacCorp, TX) was used to conduct the meta-analysis, NMA, subgroup analyses, and heterogeneity estimates.

Subgroup analysis: To avoid the potential for over-estimating treatment effects of sound therapy with too broad a definition, we differed from the previous study by stratifying studies into nine kinds of sound. In addition, the definition of customized music therapy is too broad, so we classified music therapy which formulated according to the characteristics of patients' tinnitus site into the CM group, and defined the therapy that use the pure tone which has some frequencies mixing point masking into the MPT group, such as Phase-Shift Sound and Mix pure tone. Moreover, therapies that combined CBT with any form of audiological intervention were excluded to avoid confounders, such as tinnitus retraining therapy.

Sensibility analysis: STATA (StatacCorp, TX) was used to conduct the meta-analysis, NMA, subgroup analyses, and heterogeneity estimates.

Countries involved: All the countries involved.

Keywords: Sound therapy; Customized sound; Tinnitus; A Bayesian analysis. 